\title{
8. Zusammenfassung: Methodisches Vorgehen
}

Die subjektiven Perspektiven von sieben Müttern und ihren jugendlichen Söhnen wurden unabhängig voneinander in episodischen Interviews erhoben. Durch den unmittelbaren Kontakt zu Familien, in denen ein Problem mit der Onlinespiele-Nutzung wahrgenommen wird, konnten qualitative Daten gewonnen werden, die bei der Beantwortung der Forschungsfrage Gegenstandsnähe versprechen. Die qualitative Konzeption der Studie ermöglichte es somit im Gegensatz zu quantitativen Verfahren, ohne feststehende Definitionen an den Forschungsgegenstand der exzessiven, problematischen oder pathologischen Mediennutzung heranzutreten. Es ist nicht nur die gegenstandsverankerte Vorgehensweise in der Grounded Theory, die mit dem Forschungsinteresse übereinstimmt, sondern genauso der Fokus auf Interaktionen sowie das prozessorientierte Denken. Durch die Methode des permanenten Vergleichs ließen sich die Aussagen der Mütter und Söhne familienintern und -übergreifend in Beziehung setzen und relevante Kategorien gegenstandsverankert benennen, gewichten und ausdifferenzieren. Das prozessorientierte Denken machte es möglich, die aufeinander bezogenen Interaktionen im Verlauf zu analysieren.

Der Methodenstil der Grounded Theory ist durch die Zirkularität des Erhebungs- und Auswertungsverfahrens, durch die Vorläufigkeit der Ergebnisse und ihre permanente Diskussion sowie die Dokumentation des Forschungsprozesses in Memos gekennzeichnet. Dieses Verfahren ist sehr praxisnah angelegt und ermöglicht eine durchgehende Offenheit gegenüber Neuentdeckungen und Revisionen. Die Entwicklung der gegenstandsbezogenen Theorie geschah somit durch Rückgriffe sowie durch das Verwerfen und Verfeinern der Konzepte und Kategorien. Die Auseinandersetzung mit externen Theorien erfolgte parallel zur empirischen Arbeit und erwies sich für die Anschlussfähigkeit der Ergebnisse an den Forschungsstand sowie für die Erhöhung der eigenen theoretischen Sensibilität als unverzichtbar. Das Sampling wurde schon zu Beginn der Studie auf die Befragung von Müttern und Söhnen begrenzt, um die Überschaubarkeit des Forschungsfeldes zu gewährleisten. Durch das detailgenaue Verfahren und die tief gehende Analyse konnte eine theoretische Sättigung nicht erreicht werden. Die Daten erwiesen sich jedoch als sehr ertragreich, sodass zugunsten der Komplexität nur eine geringe Anzahl an Familien befragt wurde. Aus den dargestellten Gründen wurden außerdem zwei der Familien in der Ergebnisdarstellung zurückgestellt. 